very similar, yet the $\mathrm{Al}_{1}$ atoms approach unusually close to the $\mathrm{Cr}$ atoms, whilst the $\mathrm{Al}_{2}$ atoms remain at a more normal distance. Similar relationships between electron deficiencies and shortened transition metalaluminium interatomic distances have been noted in $\mathrm{Mn}_{3} \mathrm{SiAl}_{9}$ and in $\mathrm{MnAl}_{6}$ and in both these cases it has been possible to give more reliable evidence for absorption of electrons into the vacancies in the $3 d$ shells of the transitional metal atoms.

\section{Conclusion}

The structure of $\alpha(\mathrm{AlCrSi})$ is not very convenient for assessing directly whether or not electron absorption into the transitional metal atoms takes place, but electron counts suggest that there is some limited absorption by the $\mathrm{Cr}$ atoms. Moreover, the form of the first prominent Brillouin zone is consistent with the supposition that $\alpha(\mathrm{AlCrSi})$ is an electron compound, in which the transitional metal atoms prevent valency electrons of other atoms from behaving as if they were free electrons. The shortening of some interatomic distances, mentioned above, s'ggests that some form of bonding other than that normally encountered in metallic structures is taking place in isolated regions of structures of this type, i.e. some of the bonds appear to have an ionic character.
It is a pleasure to thank Prof. Sir Lawrence Bragg and $\mathrm{Dr}$ W.H. Taylor for the provision of facilities and for their interest in the progress of this work. For financial assistance I wish to thank the Ministry of Supply (Basic Properties of Metals Committee) and, later, the Royal Commissioners for the Exhibition of 1851.

\section{References}

International Tables for X-ray Crystallography (1952). Birmingham: Kynoch Press.

Jones, H. (1934). Proc. Roy. Soc. A, 144, 225.

Mondolfo, L. F. (1943). Metallography of Aluminium Alloys. Wiley: New York.

Nelson, J. B. \& Rmey, D. P. (1945). Proc. Phys. Soc. 57,160 .

Nrcol, A. D. I. (1953). Acta Cryst. 6, 285.

Pratt, J. N. \& Raynor, G. V. (1951). J. Inst. Met. 79, 211.

RAYNOR, G. V. (1944). J. Inst. Met. 70, 531.

Raynor, G. V. \& Waldron, M. B. (1949). Phil. Mag. (7), 40, 198.

Robinson, K. (1951). Ph.D. dissertation, University of Cambridge.

Robinson, K. (1952a). Acta Cryst. 5, 397.

Robinson, K. (1952b). Phil. Mag. (7), 43, 775.

Robinson, K. (1953). Acta Cryst. 6, 667.

Acta Cryst. (1953). 6, 859

\title{
Polarization Dichroism, Form Birefringence, and Molecular Orientation in Crystalline Haemoglobins
}

\author{
By M. F. Perutz \\ Medical Research Council Unit for the Study of the Molecular Structure of Biological Systems, \\ Cavendish Laboratory and Molteno Institute, University of Cambridge, England
}

(Received 21 October 1952 and in revised form 8 July 1953)

The three principal absorption coefficients of the oxyhaemoglobin molecule were determined at $\lambda=5800 \AA$, using crystals of horse haemoglobin in which the molecules lie on lattice points. The coefficients are defined by an ellipsoid of revolution with an axial ratio of $2 \cdot 6( \pm 0 \cdot 1)$, the short axis of the ellipsoid enclosing an angle of $10^{\circ}\left( \pm 1^{\circ}\right)$ with the long axis of the molecule. This result was used to find the orientation of the molecules in two forms of human haemoglobin.

A comparative study of the molecular dichroism of different haemoglobin derivatives showed that oxy-, carboxy- and methaemoglobin and the addition compounds of methaemoglobin with azide and imidazole all have, within the limits of error, the same dichroic ratio of $2 \cdot 6$. Reduced haemoglobin alone has a dichroic ratio of 4.5. A similar difference was found between the dichroic ratios of reduced and metmyoglobin.

The birefringence of wet crystals of horse methaemoglobin changes with the refractive index of the suspension medium. The crystals were found to possess a strong negative intrinsic birefringence, which is compensated and masked by a positive form birefringence. $n_{\alpha}$ (intrinsic) is parallel to the direction of least absorption. $n_{\gamma}$ (form) encloses a small angle with the long axis of the haemoglobin molecules.

\section{Introduction}

Bragg \& Perutz (1952a) are developing a direct method of analysis of haemoglobin which depends on the plotting of the molecular Fourier transform in reci- procal space. By swelling and shrinkage of crystals of horse methaemoglobin the values of $|F|_{h o l}$ can be found at a large number of points along lines of constant $h$ and the nodes and loops of the molecular 
transform can then be traced along those lines (Fig. 1). Horse methaemoglobin alone, however, offers no possibility of finding the value of the transform between the lines and of thus establishing sign relationships between $F^{\prime}$ 's with different values of $h$.

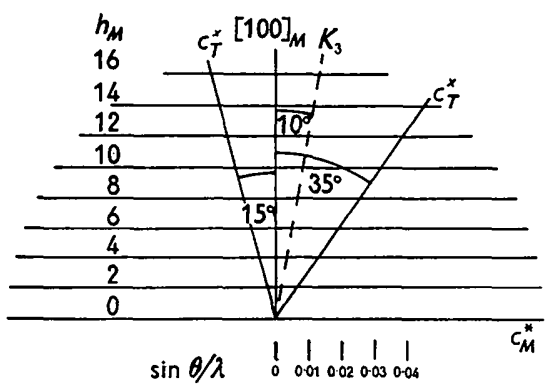

Fig. 1. Reciproealilattice plane $h 0 l$ of horse methaemoglobin showing lines of constant $h$, with two alternative directions of $c^{*}$ of tetragonal human haemoglobin superimposed.

This could be: done, and the number of points where $|F|$ can be measured could be much increased, if the reciprocal lattices of different crystalline forms of haemoglobin could be superimposed. For this to be possible three conditions must be fulfilled: first we must be sure that the molecular structure of these different forms of haemoglobin is the same, at least at the resolution of about $7 \AA$ used in this work; secondly the space group must be favourable; and thirdly methods must be available for finding the co-ordinates and orientation of the molecules in the different lattices. Recent work, to be described elsewhere, has suggested that the haemoglobins of several different mammals are similar in structure so that the first condition is probably satisfied. This paper describes how the orientation of the haemoglobin molecules in two forms of human haemoglobin can be determined by making use of the dichroic properties of the crystals. In addition to pursuing this primary objective, the optical properties of haemoglobin crystals and of their various derivatives were studied in some detail, and an interesting change in dichroism was found to accompany oxygenation and reduction.

\section{Technique}

Crystals were examined in the wet state, suspended in appropriate buffer solutions. Absorption was measured by matching the spectrum of a haemoglobin crystal against that of a haemoglobin solution of known concentration. The apparatus consisted of a Pointolite lamp and a Leitz polarizing microscope in which the eyepiece was replaced by a Zeiss microspectroscope. This has a lateral opening and a $45^{\circ}$ prism; a second beam of light could thus be projected on to the main prism and its spectrum viewed side by side with that of the light beam coming through the microscope. This second beam was made to pass a wedge-shaped trough which was filled with a haemoglobin solution.
By shifting the trough backwards and forwards in front of the hole, the thickness of solution through which the second beam passed could be varied until its spectrum exactly matched that of the crystal. The same arrangement, used without polarized light, has been described in detail and illustrated by Keilin \& Hartree (1946). Dichroic ratios could be measured with an accuracy of about $\pm 5 \%$. Absolute absorption coefficients, on the other hand, could not be measured with an accuracy better than $20 \%$, because of errors involved in the measurement of crystal thickness.

\section{Horse oxy- or methaemoglobin}

Space group $C 2 ; n=2$. The molecules lie on lattice points. Bragg \& Perutz (1952b) showed that as a first approximation their external form can be represented as an ellipsoid with axes of $54 \times 54 \times 65 \AA$, the long axis of the ellipsoid enclosing an angle of about $20^{\circ}$ with the crystallographic $a$ axis (Fig. 2). The position of the molecules on lattice points implies that the absorption coefficients of the crystal can be used to calculate directly those of the molecules themselves. Once the molecular absorption coefficients are known, they can be used to find the orientation of the molecules in other crystalline forms. (This argument would be invalidated if the dichroism of the crystals were influenced by electrical interaction between the coloured groups belonging to neighbouring molecules. Fortunately the dimensions of these groups are so small compared with the lattice constants that this effect can probably be neglected. The absence of such interaction is supported by the exact correspondence of the absorption spectra of crystals and solutions.)

Qualitative measurements indicated that the crystals exhibit dichroism of the negative uniaxial type with the direction of least absorption approximately parallel to the long axis of the molecules (Perutz, 1939). Optical absorption is not a property which necessarily follows a second-order tensor law, but crystals which are transparent in moderate thickness follow it rather closely (Hartshorne \& Stuart, 1950). Several fresh crystals of great perfection and optimum thickness showed a mean D.R. of $\mathbf{2} \cdot 6$ for light incident normal to (001), the direction of high absorption being parallel to [010]. For light parallel to [010] the same dichroic

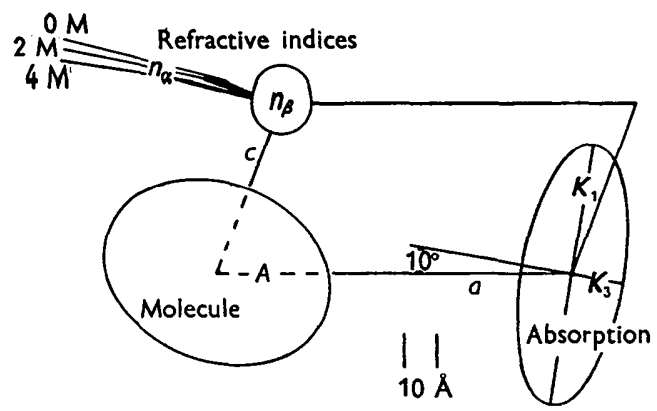

Fig. 2. Molecular orientation and crystal optics in relation to the unit cell of horse methaemoglobin. 
ratio was found, the direction of least absorption enclosing an angle of $10^{\circ}$ with [100]. Fig. 2 shows the orientation of the absorption ellipsoid relative to the unit cell and to the haemoglobin molecules. Form dichroism seems to play no part since both the dichroic ratio and the angle between [100] and $K_{3}$ were the same in salt-free crystals and in crystals suspended in $4 \mathrm{M}\left(\mathrm{NH}_{4}\right)_{2} \mathrm{SO}_{4}$ solution.

Absolute absorption coefficients of oxyhaemoglobin crystals were measured for $\lambda=5800 \AA$, where oxyhaemoglobin has a specific absorption coefficient $K_{s}=710$ (absorption coefficient of $1 \mathrm{~cm}$. of solution containing $1 \mathrm{~g}$. haemoglobin per $\mathrm{cm} .{ }^{3}$ ). The results were $K_{1}=K_{2}=690 ; K_{3}=260$, giving a mean absorption coefficient $K_{m}=550$. The wet crystals contain $63.5 \%$ haemoglobin, give a calculated $K_{m}$ of 450 . The discrepancy between the calculated and observed values of $K_{m}$ is undoubtedly due to errors in the measurement of crystal thickness; when corrected for this the absorption coefficients of the crystals would be $K_{1}=K_{2}=565, K_{3}=210$.

\section{Human oxy - or methaemoglobin}

Space group $P 4_{1} 2_{1} ; n=4$. The symmetry elements of the space group are shown in Fig. 3. The molecules lie in special positions on the dyads and, as Bragg \& Perutz (1952c) have shown, their $A$ axes are likely to be tilted so as to enclose a small angle with the tetrad; this is indicated by the arrows in Fig. 3. If

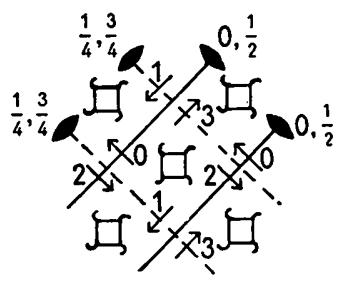

Fig. 3. Space group symmetry of human oxyhaemoglobin $\left(P 4_{1} 2_{1}\right)$. The numbers refer to molecules at $0, \frac{1}{4}, \frac{2}{4}, \frac{3}{4}$, and the arrows indicate the direction of tilt.

all the molecules were oriented with $K_{3}$ parallel to the tetrad, the dichroic ratio of the crystals should be $2 \cdot 6$, the same as in horse methaemoglobin. In fact D.R. $=1 \cdot 9( \pm 0 \cdot 1)$, which shows that $K_{3}$ must be inclined to the tetrad. The angle of inclination can be calculated from the molecular dichroism and the space-group symmetry, and is found to be $25^{\circ}\left( \pm 4^{\circ}\right)$. The method of calculation is given in an appendix.

Since $K_{3}$ encloses an angle of $10^{\circ}$ with $A$ (see Fig.2) this result leads to two possible orientations of the molecule with angles $A:[001]_{T}=-15^{\circ}$ or $+35^{\circ}$.

Superposition of the reciprocal lattices of monoclinic horse haemoglobin $(M)$ and tetragonal human haemoglobin $(T)$ can now be considered. Since lattice $M$ contains only one molecule per lattice point $|F|_{h o l}$ is determined by the magnitude of the molecular structure factor alone and is not modified by inter- ference between the different molecules in the unit cell. In lattice $T$ this is true only of $|F|_{00 t}$; therefore the $c^{*}$ axis is the only part of reciprocal lattice $T$ which can be directly superimposed on the reciprocal lattice $M$. Since $c_{T}^{*}$ is normal to the dyad axes of the molecules it must lie in the $(h 0 l)_{M}$ plane. Its orientation in that plane is determined by the angles $c: K_{3}=25^{\circ}$ and $c: A=-15^{\circ}$ or $+35^{\circ}$, and is shown in Fig. 1 .

\section{Human reduced haemoglobin}

Space group $P 2_{1} ; n=2$. The molecules lie in general positions, but Patterson projections indicate that the unit cell is approximately body-centred and that the molecules are oriented with $A \|[100]$ and $B \|[010]$ (Perutz, Howells \& Trotter, unpublished results). The crystals show negative dichroism and the mean D.R. is $4.5( \pm 0.2)$; the direction of minimum absorption is approximately parallel to [100]. Accurate determination of this direction is prevented by an unfavorable crystal habit.

It was a surprising discovery to find the D.R. of human reduced haemoglobin to be greater, by a factor of 1.7 , than the D.R. of horse methaemoglobin, in which the molecules are known to be strictly parallel. It was suspected that the reduced form may have an intrinsically higher D.R. than oxy- or methaemoglobin, and the following experiment was devised to test this hypothesis. Oxy-, carboxy- and methaemoglobin on the one hand, and reduced haemoglobin on the other hand, always crystallize in different forms. It had previously been found that crystals of oxyhaemoglobin could not be reduced, nor crystals of the reduced form oxygenated, without destruction of the crystal lattice. It seemed that this difficulty might be overcome by keeping the crystals in a buffer which is so concentrated that a break-up of the lattice is prevented by the complete insolubility of the protein.

Crystals of reduced haemoglobin were therefore suspended in $3 \mathrm{M}$ phosphate buffer of $p_{\mathrm{H}} 6 \cdot 8$, in which all forms of human haemoglobin are insoluble. They were then transformed into oxy-, carboxy- or methaemoglobin by exposing them to air or carbon monoxide or by treating them with ferricyanide. Such treatment was found to reduce the D.R. to $2 \cdot 6,2 \cdot 5$ and 2.7 respectively. All these values are the same, within the experimental error, as the D.R. of horse methaemoglobin; except for the development of some cracks, the crystals remained intact during this treatment, the change in D.R. being due, apparently, to a molecular transformation within the crystal lattice. The chemical significance of this result will be discussed elsewhere.

The orientation of the molecules will now be considered. If experimental errors could be neglected, the value of D.R. $=2 \cdot 6$ with minimum absorption $\|[100]$ would imply $K_{3}||[100]$ and $A:[100]=10^{\circ}$. However, the error in the determination of D.R. is of the order of $5 \%$, which means that the two molecules in the 
unit cell could be inclined to each other by an angle $\leq 30^{\circ}$ without causing a detectable change in the D.R. Hence the conclusion allowed by the optical results is merely that $K_{3}:[100] \leq 15^{\circ}$, whence $A:[100] \leq 25^{\circ}$. This does not add to the information already provided by the Patterson projections, but merely confirms it in a general way.

\section{Dichroic ratios of other haemoglobin derivatives and of myoglobin}

Since the D.R. of reduced haemoglobin crystals falls on oxidation to methaemoglobin it was of interest to see whether that of methaemoglobin crystals rises on reduction. Crystals of horse methaemoglobin were suspended in $3.5 \mathrm{M}\left(\mathrm{NH}_{4}\right) \mathrm{SO}_{4}$, buffered to $\mathrm{p}_{\mathrm{H}} 7$, and a small quantity of sodium hydrosulfite was added. This caused a rise in D.R. from $2 \cdot 6$ to $3 \cdot 6$. The maximum of the absorption band was at $5700 \AA$, whereas the normal position of that band is at $5500 \AA$. On dissolving the crystals the band shifted to the normal position. The comparatively low D.R. and the abnormal position of the absorption band indicate that the reduced haemoglobin formed in these crystals is in some way abnormal, and that the formation of the proper compound is prevented by the lattice forces.

A similar experiment was tried with crystals of horse metmyoglobin, kindly lent to me by Dr J. C. Kendrew. These crystals have the space group $P 2_{1} 2_{1} 2_{1}$ with four molecules in the unit cell, which are known from Patterson projections to be non-parallel (Kendrew \& Trotter, 1953). The crystals show dichroism of the negative uniaxial type with a D.R. $=1 \cdot 7$. On reduction with sodium hydrosulfite this rises to $2 \cdot 4$. The absorption spectrum of the reduced crystals is identical with that of the corresponding solution.

Methaemoglobin forms a number of addition compounds, all recognizable by their characteristic absorption spectra. In view of the great change in D.R. on reduction it was of interest to find out whether a similar change would be produced by the formation of one of these compounds. The compounds were made by allowing the appropriate substances to diffuse into methaemoglobin crystals suspended in $3.5 \mathrm{M}\left(\mathrm{NH}_{4}\right)_{2} \mathrm{SO}_{4}$. The results showed that both azide and imidazole methaemoglobin have the same D.R., within the limits of the experimental error, as methaemoglobin itself. Examination of fluoride methaemoglobin and of alkaline methaemoglobin failed, because the absorp. tion spectra of these compounds in the crystal were different from those in solution. The chemical significance of these results will be discussed elsewhere.

\section{Form birefringence of horse methaemoglobin}

The birefringence of haemoglobin crystals is due to the superposition of three effects: the intrinsic birefringence of the haem groups, that of the globin, and the form birefringence of the entire molecule. Perutz
\& Mitchison (1950) showed that the intrinsic birefringence is subject to strong dispersion and rises to a maximum on the long-wave side of each absorption band. However, in thick crystals only red light is transmitted so that measurements of birefringence of such crystals are confined to a spectral region around $0 \cdot 6 \mu$. Boyes-Watson, Davidson \& Perutz (1947) measured the birefringence of a thick salt-free crystal of methaemoglobin in three mutually perpendicular planes and found $n_{\beta}-n_{\alpha}=0.0018, n_{\gamma}-n_{\alpha}=0.0070$, $\alpha:[100]=16^{\circ}, \gamma:[001]=5^{\circ}$.

In the present study an attempt was made to determine the form birefringence. Wet crystals mounted between flats in liquid paraffin were examined on a Fedorov stage. A Pointolite lamp and an interference filter transmitting at $\lambda=6790 \AA$ were used as a light source. $n_{\gamma}-n_{\alpha}$ was determined by tilting the crystals on the Fedorov stage until [010] was parallel to the microscope axis. In this orientation the (110) faces form a convenient pair of wedges which produce interference fringes between the two rays vibrating parallel to the $\bar{X}$ and $Z$ axes of the indicatrix. The distance between the fringes, which is directly related to $n_{y}-n_{\alpha}$, can be measured with an accuracy of $2-4 \%$, depending on their number. The optic axial angle $2 \mathrm{~V}$ was measured directly by tilting the crystals in the optic axial plane until extinction was observed. The measured angle between the two extinction positions was corrected for refraction and was accurate to about $2 \% \cdot n_{\beta}-n_{\alpha}$ was calculated from $n_{\gamma}-n_{\alpha}$ and $2 \mathrm{~V}$.

Using these methods, the birefringence of horse methaemoglobin crystals was determined in saltfree medium, in $2 \mathrm{M}$ and in $4 \mathrm{M}$ ammonium sulphate solution. At this stage a serious difficulty was encountered. Saltfree haemoglobin crystals cannot be transferred to a salty medium without breaking up, and saltfree and salty crystals therefore have to be grown independently by different methods. The birefringence of different crops of crystals, however, was found to differ according to the state of the pigment, and these differences were found to be as great as the changes due to form birefringence. In the circumstances a comparison of salty and saltfree crystals proved to be meaningless, and all that could be done was measurement of the change of birefringence which resulted when crystals were transferred from $2 \mathrm{M}$ to $4 \mathrm{M}$ ammonium sulphate solution.

The results are shown in Table 1. An increase in the refractive index of the suspension medium causes $n_{y}-n_{\alpha}$ and $2 V$ to rise. If the refractive index of the suspension medium could be raised until it equalled that of the dry protein (which is of the order of 1.6), a negative intrinsic birefringence would be found with the acute bisectrix $\left(n_{\alpha}\right)$ approximately parallel to the direction of least absorption $\left(K_{3}\right)$. This confirms an impression gained during Perutz \& Mitchison's study of dispersion, i.e. that the intrinsic birefringence of haemoglobin is largely dominated by the contribution of the haem groups and has the same sign and 
Table 1

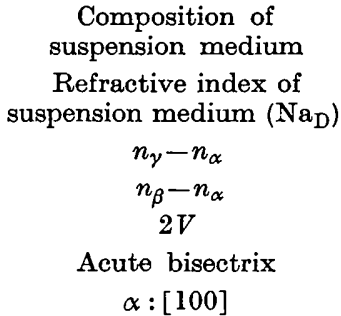

Composition of suspension medium

Refractive index of $n_{\gamma}-n_{\alpha}$

$n_{\beta}-n_{\alpha}$

ute bisectrix

$\alpha:[100]$

$2 \mathrm{M}\left(\mathrm{NH}_{4}\right)_{2} \mathrm{SO}_{4}$
$1 \cdot 3695$
0.0063
$0 \cdot 0020$
$69^{\circ}$
$\gamma$
$11^{\circ}-12^{\circ}$

Note: $\beta \|[010]$.

$\begin{array}{cc}4 \mathrm{M}\left(\mathrm{NH}_{4}\right) \mathrm{SO}_{4} & \text { Difference } \\ 1 \cdot 397 & \\ 0.0071 & +0.0008 \\ 0.0028 & +0.0008 \\ 78^{\circ} & +9^{\circ} \\ \gamma & \\ 8^{\circ}-9^{\circ} & -3^{\circ}\end{array}$

the dichroic ratio to fall to the value of $2 \cdot 6$ observed in methaemoglobin of horse. The direction of least absorption is approximately parallel to [100], which shows that the long axes of the two molecules in the unit cell must lie approximately along that direction.

4. Of the various haemoglobin derivatives examined, acid methaemoglobin, its addition compounds with imidazole and azide, and oxy- and carboxyhaemoglobin all have within the limits of error the same molecular dichroism, with a dichroic ratio of $2 \cdot 6$. When crystals of horse methaemoglobin are reduced, their dichroic ratio rises from $2 \cdot 6$ to $3 \cdot 6$, and when metmyoglobin crystals are reduced, it rises from $1 \cdot 7$ to $2 \cdot 4$.

5. Horse methaemoglobin crystals were found to possess a strong negative intrinsic birefringence which is compensated and masked by a positive form birefringence. The intrinsic refractive-index ellipsoid has the same sign and orientation as the absorption ellipsoid; $n_{\alpha}$ (intrinsic) is parallel to $K_{3}$, the axis of least absorption. It appears, therefore, that the intrinsic birefringence is dominated by the coloured haem groups. The form birefringence is uniaxial and positive, with the optic axis of the refractive index ellipsoid approximately parallel to the long axis of the haemoglobin molecule (Bragg \& Perutz, 1952b).

\section{APPENDIX I}

\section{Relation between dichroic ratio and molecular orientation in tetragonal human haemoglobin}

For space group symmetry see Fig. 3. Molecular absorption coefficients: $K_{1}=K_{2} ; K_{3}$. Absorption coefficients of tetragonal crystals: $\chi_{1}=\chi_{2} ; \chi_{3}$.

Both the $K$ 's and the $\chi^{\prime}$ 's define ellipsoids of revolution. $\operatorname{Sin}\left(K_{3}: \chi_{3}\right)=s_{33} ; \cos \left(K_{3}: \chi_{3}\right)=c_{33}$.

Let $\chi_{1}$ and $\chi_{2}$ be parallel to the dyads, so that $K_{2} \| \chi_{1}$ for one pair of molecules and $K_{2} \| \chi_{2}$ for the other pair. The tensor between the $K$ 's and the $\chi^{\prime}$ 's then takes the following form: between the tetrad and $A$, the so-alled chain direc tion which is parallel to $[100]$ in horse methaemoglobin.

3. Monoclinic crystals of human reduced kaemoglobin show negative uniaxial dichroism with a dichroic ratio of $4 \cdot 5( \pm 0 \cdot 2)$. When such crystals are suspended in concentrated buffer solutions and treated with air, carbon monoxide or ferricyanide, they are transformed into oxy-, carboxy- or methaemoglobin without change of external form. Such treatment causes

$\begin{array}{ccccc} & & \chi_{1} & \chi_{2} & \chi_{3} \\ \text { Molecules 0 and 2 } & K_{1} & c_{33} & 0 & \pm s_{33} \\ & K_{2} & 0 & 1 & 0 \\ & K_{3} & \pm s_{33} & 0 & c_{33} \\ \text { Molecules 1 and 3 } & K_{1} & 0 & c_{23} & 0 \\ & K_{3} & 1 & 0 & \pm s_{33} \\ & K_{3} & 0 & \pm s_{33} & c_{38}\end{array}$


It can be shown to follow that

$$
\begin{aligned}
\chi_{1}=\chi_{2} & =2\left[K_{1}\left(1+c^{2}\right)+K_{3}\left(1-c^{2}\right)\right], \\
\chi_{3} & =4\left[K_{3} c^{2}+K_{1}\left(1-c^{2}\right)\right] .
\end{aligned}
$$

For the measured values $\chi_{1} / \chi_{3}=1.9$ and $K_{1} / K_{3}=$ $2 \cdot 6$, this gives an angle $K_{3}: \chi_{3}=25^{\circ}$. Errors of $0 \cdot 1$ in the measured dichroic ratios $\chi_{1} / \chi_{3}$ and $K_{1} / K_{3}$ lead to errors in the angle of $3^{\circ}$ and $1^{\circ}$ respectively, so that the maximum error in the estimate of this angle should be less than $4^{\circ}$.

\section{APPENDIX II}

\section{Sources of experimental error}

\section{(a) Aperture of objective}

The objectives used in this work had numerical apertures ranging from $0 \cdot 21$ to $0 \cdot 65$, corresponding to angles of incidence ranging from $6^{\circ}$ to $19^{\circ}$. All the crystals studied showed uniaxial dichroism. Hence, in the position where the electric vector is parallel to the equatorial plane of the absorption ellipsoid, the absorption coefficient is independent of the angle of incidence. If the electric vector is normal to the equatorial plane the absorption coefficient does vary with the angle of incidence, but in fact the variation of $K$ within $20^{\circ}$ of $K_{3}$ is so small that no measurable error could be introduced by using an objective of N.A. $\leq 0.65$.

\section{(b) Age of crystals}

The mean dichroic ratio of a fresh crop of horse methaemoglobin crystals was $2 \cdot 6$. Crystals which had been kept for a year or more gave variable readings below this value, some of them as low as $2 \cdot 1$. The mean absolute absorption coefficient $K_{m}$ of old crystals was only $60 \%$ of the calculated one, which shows that the absorption spectrum of some or of all the molecules must have undergone some change through denaturation, although the crystals were otherwise indistinguishable from fresh ones. When the crystals were suspended in $0.1 \mathrm{M}$ phosphate buffer they dissolved only very slowly, but nevertheless completely. The solution could be reduced with hydrosulfite and recombined with carbon monoxide, and both these derivatives showed normal absorption spectra. Although the signs of denaturation are thus somewhat indefinite, the optical results make one doubt that proteins remain completely unchanged over long periods, even if kept in the form of crystals suspended in concentrated buffer solutions. The variations in the birefringence of different crystal crops point to the same conclusion.

I should like to thank Sir Lawrence Bragg and Dr J.C. Kendrew for their helpful criticism in the interpretation of the results.

\section{References}

Boyes-Watson, J., Datidson, E. \& Perutz, M. F. (1947). Proc. Roy. Soc. A, 191, 83.

Bragg, W. L. \& Perutz, M. F. (1952a). Proc. Roy. Soc. A, 213, 425 .

BragG, W. L. \& Perutz, M. F. (1952b). Acta Cryst. 5, 277.

BragG, W. L. \& Perutz, M. F. (1952c). Acta Cryst. 5, 323.

Hartshorne, N. H. \& Stuart, A. (1950). Crystals and the Polarising Microscope, 2nd ed. London: Arnold.

KeILIN, D. \& Hartree, E. F. (1946). Nature, Lond. $157,210$.

Kendrew, J. C. \& Trotyer, I. F. (1953). In preparation.

Perutz, M. F. (1939). Nature, Lond. 143, 731.

Perutz, M. F. \& Mitchison, J. M. (1950). Nature, Lond. 166, 677 . 